\title{
Article \\ Deciphering Bacterial Community of the Fallow and Paddy Soil Focusing on Possible Biocontrol Agents
}

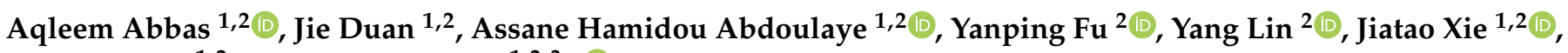 \\ Jiasen Cheng ${ }^{1,2}$ and Daohong Jiang $1,2,3, *$ (i)
}

1 State Key Laboratory of Agriculture Microbiology, Huazhong Agricultural University, Wuhan 430070, China; aqlpath@gmail.com (A.A.); $18404968698 @ 163 . c o m(J . D$.$) ; doul_as@yahoo.fr (A.H.A.);$ jiataoxie@mail.hzau.edu.cn (J.X.); jiasencheng@mail.hzau.edu.cn (J.C.)

2 Hubei Key Laboratory of Plant Pathology, Huazhong Agricultural University, Wuhan 430070, China; yanpingfu@mail.hzau.edu.cn (Y.F.); yanglin@mail.hzau.edu.cn (Y.L.)

3 Hubei Hongshan Laboratory, Wuhan 430070, China

* Correspondence: daohongjiang@mail.hzau.edu.cn

check for

updates

Citation: Abbas, A.; Duan, J.;

Abdoulaye, A.H.; Fu, Y.; Lin, Y.;

Xie, J.; Cheng, J.; Jiang, D.

Deciphering Bacterial Community of

the Fallow and Paddy Soil Focusing

on Possible Biocontrol Agents.

Agronomy 2022, 12, 431.

https://doi.org/10.3390/

agronomy12020431

Academic Editor: Karen Barry

Received: 12 December 2021

Accepted: 31 January 2022

Published: 9 February 2022

Publisher's Note: MDPI stays neutral with regard to jurisdictional claims in published maps and institutional affiliations.

Copyright: (C) 2022 by the authors. Licensee MDPI, Basel, Switzerland. This article is an open access article distributed under the terms and conditions of the Creative Commons Attribution (CC BY) license (https:// creativecommons.org/licenses/by/ $4.0 /)$.

\begin{abstract}
In pursuing higher rice production, we have often jeopardized soil at an alarming rate. It is hypothesized that intensive farming practices degrade soil health and increase the abundance of rice diseases while fallowing increases the abundance of biocontrol agents. In this study, the bacterial community was monitored in the paddy and fallow soil. Proteobacteria, Bacteroidetes, and Actinobacteria were abundant in the fallow soil, whereas Acidobacteria, Chloroflexi, and Gemmatimonadetes were more abundant in the paddy soil. Among the potential biocontrol agents, Bacillus, Thiobacillus, Rhizobium, Massilia, Rhizobacter, Streptomyces, Micromonospora, and Pseudonocardia were more abundant in the fallow soil, while Pseudomonas and Burkholderia were more abundant in the paddy soil. The possible rice pathogens, i.e., Xanthomonas and Erwinia, were more abundant in the paddy soil. The alpha diversity was higher in paddy soil than in fallow soil. Additionally, the principal coordinate analysis based on UniFrac distances revealed distinct clusters of the soils. Moreover, the functional analysis suggested that the fallow soil was abundant in genes associated with the biosynthesis of siderophores and secondary metabolites. In contrast, the paddy soil was abundant in genes related to plant-pathogen interactions. In conclusion, these results highlight the significance of fallowing to improve soil health.
\end{abstract}

Keywords: biocontrol agents; paddy soil; fallow soil; diversity; ecological functions

\section{Introduction}

Rice (Oryza sativa L.) is among the most important cereal crops for global food security and is the staple food of 3.5 billion people. In the world, China is the major rice producer and represents $40 \%$ of global rice production [1]. More than as a food crop, its residues, such as husk and straw, are used as animal feed, to grow mushrooms, and to produce biofuels [2]. Soil is a complex dynamic ecosystem that hosts billions of microorganisms. These microorganisms play a significant role in maintaining soil health. Previous research has shown that soil microorganisms are influenced by plant genotypes, cropping history, soil types, environmental conditions, and other agricultural practices [3-5]. However, intensive cropping systems, double-season rice cropping systems, limited crop rotations, inappropriate management practices, and intensive usage of pesticides degrade soil health, hamper beneficial microorganisms' ecological services and functions, and jeopardize sustainable land productivity [6]. In response to intensive pesticide applications, severe plant diseases have emerged and caused substantial economic losses in rice crops, such as rice blast, rice sheath blight, rice sheath rot, and bacterial leaf blight [1,7].

Previous research shows that fallowing increases soil fertility; keeping the paddy soil fallow can enhance the abundance of beneficial soil microorganisms [8]. These beneficial 
microorganisms are biocontrol agents (BCAs) which can promote growth and yield. They can make nutrients available to rice by solubilizing phosphate and nitrogen $[9,10]$, reducing abiotic stresses [11], assisting in the improvement in the root architecture [4], and protecting crops from severe diseases. This occurs when they compete with the pathogens for nutrients and space or when they show antagonistic activities or induce resistance [12-16]. Thus, a well-planned cropping system increases the practical usage of natural resources and maintains soil fertility for a long time to gain high yields of rice crops. We hypothesized that fallow soil would have abundant biocontrol agents and fewer rice pathogens than paddy soil. To test this hypothesis, we collected soil samples from the fallow and paddy soil to study the effects of soil types using amplicon sequencing. This was based on nextgeneration sequencing (NGS) of 16S rRNA on the diversity, composition, and functions of the bacterial community, focusing on bacterial BCAs and rice pathogens.

\section{Materials and Methods}

\subsection{Soil Sample Collection and Preparation}

The present research was conducted in the research farms of Huazhong Agricultural University, Wuhan, Hubei Province, China. Soil samples were collected from a $10-15 \mathrm{~cm}$ depth from the paddy and fallow soils. The paddy fields were planted with the commonly grown rice crop, and no rice crop was grown on the fallow fields. Four biological replicates were sampled from four fields of each soil type resulting in 32 samples (Supplementary Materials: Table S1).

\subsection{Soil DNA Extraction}

Soil genomic DNA was extracted from $0.5 \mathrm{~g}$ of freeze-dried soil using the PowerSoil kit (MO BIO Laboratories, Carlsbad, CA, USA). The DNA quality was checked on $1.2 \%$ agarose gel electrophoresis, and the NanoDrop 2000 spectrophotometer (Thermo Fisher Scientific Inc., Wilmington, DE, United States) was used to determine the DNA purity and concentration. The DNA was preserved at $-80^{\circ} \mathrm{C}$ before amplicon library preparation and sequencing.

\subsection{Amplicon Library Preparation and Sequencing}

Amplicon library preparation and Illumina ${ }^{\circledR}$ MiSeq sequencing (Illumina, San Diego, CA, USA) were carried out by GENEWIZ, Inc. (Suzhou, China). The 16S rRNA, V3-V4 gene fragments were amplified by the primer pairs 338F and 806R (F: $5^{\prime}$-CCTACGGRRBGCASCA GKVRVGAAT- $3^{\prime}$ and R: $5^{\prime}$ - GGACTACNVGGGTWTCTAATC C-3') [17]. The PCR products were extracted from $2 \%$ agarose gels, purified using the AxyPrep DNA Gel Extraction Kit (Axygen Biosciences, Union City, CA, USA), and quantified using QuantiFluorTM-ST (Promega, Madison, WI, USA) according to the manufacturer's protocol. The purified amplicons were pooled in equimolar concentrations and processed with MiSeq Reagent Kit V2. Then 300 bp paired-end dual index sequencing was performed with an Illumina MiSeq instrument (Illumina, San Diego, CA, USA). Raw sequences were deposited in NCBI's BioProject database under accession number PRJNA702203.

\subsection{Bioinformatics Analysis}

Raw FASTQ files were quality-filtered and merged using Quantitative Insights Into Microbial Ecology (QIIME 2 v. 2018.11, https: / / qiime2.org/, accessed on 14 October 2021) [18]. The final outputs from the quality filtering and merging were a Feature Table containing ASVs (OTUs) and a Feature Data containing sequences associated with the ASVs [19]. Potential host plant-derived contaminants such as chloroplast and mitochondria were removed from the Feature Table and Feature Data. Taxa assignment was carried out against the SILVA SSU non-redundant database (132 release) [20]. The samples associated with fallow and paddy soil were rarefied to 16,717 sequences to ensure an even sampling depth. The rarefied feature table was then used to measure alpha and beta diversity. Alpha diversity metrics such as Observed species [21], Shannon's diversity index [22], Pielou's measure of species evenness [23], Faith's 
phylogenetic diversity [24], and Chao 1 [25] were calculated. The beta diversity metrics such as weighted and unweighted UniFrac [26] were used to test the association of microbial communities of the soil types. Principal coordinates analysis (PCoA) plots were visualized using EMPeror [27]. The relative abundance of phyla, classes, orders, and families, and all genera assumed to be possible bacterial BCAs and rice pathogens, were also calculated. The website Jvenn was used to construct Venn diagrams of the shared and unique OTUs (http:/ /jvenn.toulouse.inra.fr/app/example.html, accessed on 14 October 2021).

\subsection{Bacterial Community Function Prediction}

Phylogenetic Investigation of Communities by Reconstruction of Unobserved States (PICRUSt) [28] was used to make functional inferences. Closed reference OTU picking was performed against the Greengenes database 13.8 [29]. The OTU table was normalized based on each organism's expected 16S copy number. The normalized OTU table was then used as input to make functional predictions of Kyoto Encyclopedia of Genes and Genomes (KEGG) Ortholog (KOs). To collapse all KOs to their corresponding KEGG pathways, KOs were classified by function. By multiplying OTU abundances by the set of gene abundances for each taxon, the contribution of each OTU to a particular gene function was calculated. Functional categories such as disease suppression, molecular communication, nutrition, and other functional categories relevant to growth promotion and plant fitness were compared between fallow and paddy soil. Statistical Analysis of Metagenomic Profiles (STAMP) [30] was used to show the annotated table of projected gene family counts for each sample. At a $q$-value filter of $<0.05$, all predictions were treated to a multiple test correction using the Benjamini-Hochberg false discovery rate (FDR) [31].

\subsection{Statistical Analysis}

All experiments were performed with a minimum of three or more biological replicates. The significant differences between the relative abundance of the microbial community and their respective functional profiles of the two soil types were further explored using the STAMP v. 2.1.3 [30]. The groups with significant differences were identified using the Welch's $t$-test with multiple testing corrections using the Benjamini-Hochberg FDR. $Q$ values (Benjamini-Hochberg FDR corrected $p$-values) $<0.05$ were set as significant levels. A pairwise Kruskal-Wallis test was conducted to analyze the alpha diversity. A non-parametric Pairwise Permutational multivariate analysis of variance (PERMANOVA) pairwise test ( $\alpha=0.05$ ) [32] was performed on the beta diversity metrics to analyze the difference in microbial composition at soil types. Figures and tables were reconstructed using GraphPad Prism Software (San Diego, CA, USA) v. 8.0.1, Microsoft PowerPoint and Microsoft Excel 2019.

\section{Results}

\subsection{Sequencing Results and Quality Control}

There were a total of 1,876,081 raw reads across the paddy and fallow soil input libraries. After quality filtering and removal of the host plant, Protista DNA, and other non-target DNAs, 988,354 reads were retained (Supplementary Materials: Table S1).

\subsection{Analysis of Diversity of the Microbial Community of the Fallow and Paddy Soil \\ 3.2.1. Rarefaction Curves Ensure an Even Sampling Depth}

The samples associated with the paddy and fallow soil were rarefied to 16,717 sequences to ensure an even sampling depth. The rarefied feature table was then used to measure alpha and beta diversity (Figure 1). The rarefaction curves of both soil types were flattened upon reaching about 2000-3500 sequencing depth, suggestive that the sequencing depth was sufficient to reliably describe the fallow and paddy soil microbial communities. 

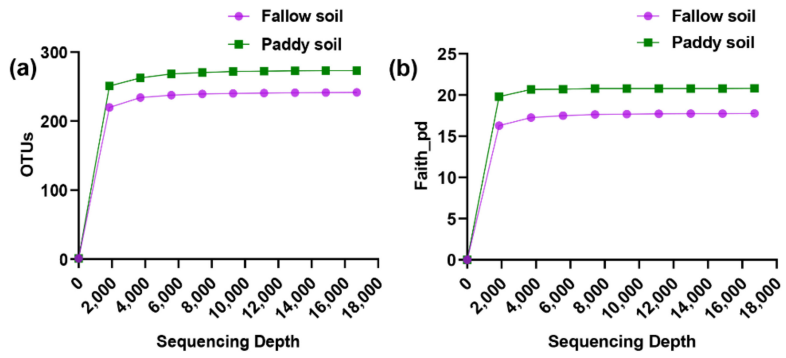

Figure 1. Rarefaction curves of soil samples associated with the fallow and paddy soil. (a) Rarefaction curve showed the observed OTUs against sequencing depth in each soil type; (b) Rarefaction curves based on phylogenetic diversity (Faith_pd). Solid circles and squares indicated the means of OTU and Faith_pd of fallow and paddy soil samples against sequencing depth.

\subsubsection{Alpha Diversity and Beta Diversity}

Chao 1, Phylogenetic diversity (faith-PD), Shannon index, Observed species, and Pielou's evenness were used to measure the alpha diversity of the bacterial communities of the fallow and paddy soils. A significant difference with $p<0.05$ for the majority of the alpha diversity indices was observed. The alpha diversity was higher in the paddy soil than in the fallow soil (Figure 2a-e). The bacterial community showed significant differences between the paddy and fallow soil according to Kruskal-Wallis (pairwise) results for the alpha diversity measurements, including Shannon index $p(0.04)<0.05$, Faith-pd $p(0.0001)<0.05$, Observed species $p(0.01)<0.05$, and Chao $1 p(0.002)<0.05$ (Supplementary Materials: Table S2), except for Pielou's evenness $p(0.5)>0.05$ (Supplementary Materials: Table S2). Principal coordinate analysis (PCoA) on the weighted and unweighted UniFrac distances showed a clear grouping between bacterial communities of the fallow and paddy soils (Figure 2f,g). Moreover, the PERMANOVA test with 999 permutations showed significant differences between soil types in the bacterial community, including weighted UniFrac $p(0.001)<0.05$ and unweighted UniFrac $p(0.001)<0.05$, respectively (Supplementary Materials: Table S3). In terms of OTUs abundance, the paddy and fallow soil samples shared 520 OTUs. Approximately 2134 OTUs were unique to the paddy soil, while 1679 OTUs were unique to the fallow soil. Higher OTUs in the paddy soil indicated higher bacterial diversity than in the fallow soil (Figure 2h).

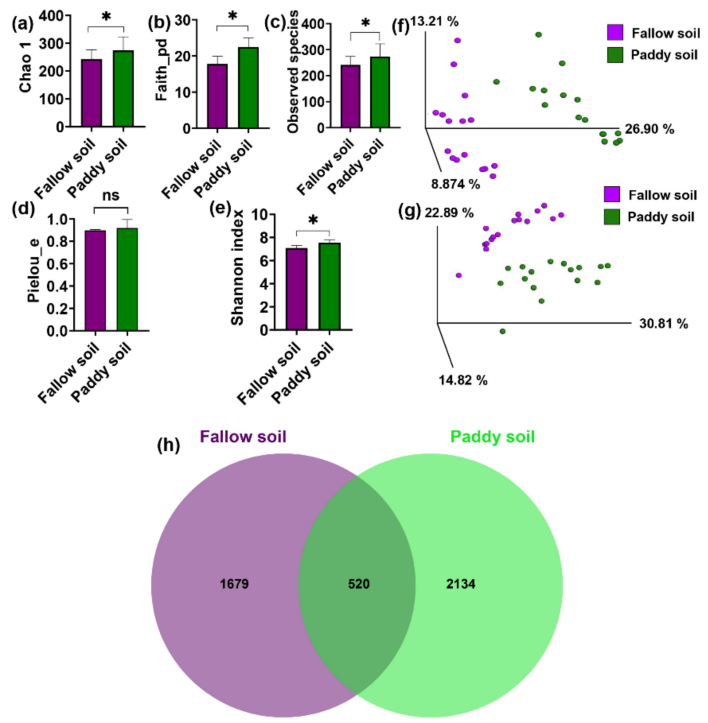

Figure 2. The $\alpha$ - and $\beta$-diversity of the bacterial community. (a) Chao 1; (b) Phylogenetic diversity; (c) OTU richness; (d) Evenness; and (e) Shannon index. Bars represent mean; error bars denote standard deviation; * (asterisks) over the bars represent significant differences $(p<0.05)$, and ns over the bars represent non-significant differences. PCoA ordinations of the bacterial community were based on the Unweighted UniFrac distance (f) and Weighted UniFrac distance (g). PERMANOVA determined differences in bacterial beta diversity of the two kinds of soil, (h) Unique and shared OTUs in the two soil types. 


\subsection{Microbial Community Composition}

3.3.1. Bacterial Community Composition at Phyla, Class, and Order Level

The phyla Acidobacteria, Actinobacteria, Armatimonadetes, Bacteroidetes, Chloroflexi, Firmicutes, Gemmatimonadetes, Latescibacteria, Nitrospinae, Nitrospirae, Patescibacteria, Proteobacteria, Verrucomicrobia, Deinococcus-Thermus, Elusimicrobia, Fibrobacteres, Rokubacteria, Zixibacteria, Planctomycetes, and others were detected in the paddy and fallow soil (Figure 3a). Among these phyla, the phylum Proteobacteria largely dominated in both the fallow and paddy soil accounting for $52 \%$ and $45 \%$ relative abundance, respectively. The relative abundance of phyla Bacteroidetes and Proteobacteria was significantly higher in the fallow soil than in the paddy soil $(p<0.05)$ (Figure $3 a)$. The relative abundance of Actinobacteria was also higher in the fallow soil than in the paddy soil. Conversely, the relative abundance of Gemmatimonadetes, Chloroflexi, and Acidobacteria was significantly higher in the paddy soil $(p<0.05)$ (Figure 3a). The relative abundance of dominant phyla Bacteroidetes, Actinobacteria, Patescibacteria, Acidobacteria, and Gemmatimonadetes in the fallow soil was $17.5 \%, 13.4 \%, 12.0 \%, 1.5 \%$, and $1.3 \%$, respectively (Figure 3a). However, in the paddy soil the relative abundance of Patescibacteria, Bacteroidetes, Actinobacteria, Gemmatimonadetes, Chloroflexi, and Acidobacteria was 12.4\%, 12.3\%, 11.5\%, 6.0\%, $5.7 \%$, and $5.0 \%$, respectively (Figure 3a). The relative abundance of Deinococcus-Thermus, Elusimicrobia, Fibrobacteres, Rokubacteria, Zixibacteria, and Planctomycetes was very low (0.1) in both soil types (Figure 3a). Deinococcus-Thermus and Rokubacteria were more abundant in the fallow soil; however, phyla Elusimicrobia, Fibrobacteres Zixibacteria, and Planctomycetes were more abundant in the paddy soil (Figure 3a).

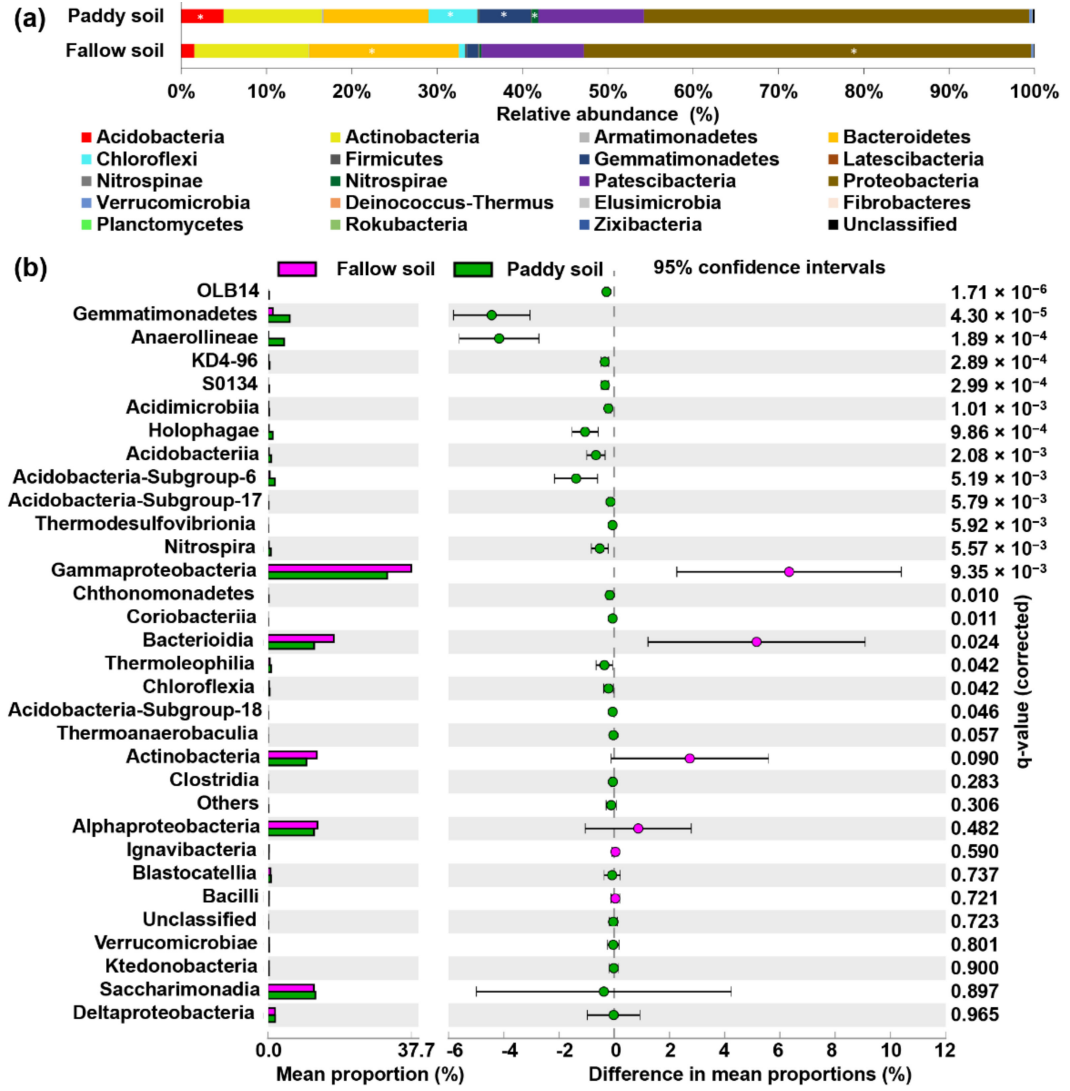

Figure 3. Relative abundance of bacterial phyla and classes detected in the fallow and paddy soil. (a) Dominant phyla with the relative abundance in both soil types, asterisk indicate relative abundance of the phyla is significantly higher in that type of soil; (b) Relative abundance of bacterial classes in both soil types, determined by Welch's $t$-test with a Benjamini-Hochberg FDR test correction to calculate $q$-values. 
Similarly, among the 32 classes with relative abundance $0.5 \%$ or higher, 26 were significantly more abundant in the paddy soil, and six were significantly more abundant in the fallow soil (Figure 3b). For example, the most abundant classes in the fallow soil were Gammaproteobacteria, Bacteroidia, Alphaproteobacteria, Actinobacteria, Saccharimonadia, and Deltaproteobacteria with relative abundances $37.63 \%, 17.30 \%, 12.97 \%, 12.81 \%$, and $1.88 \%$ (Figure $3 \mathrm{~b}$ ). In contrast, the most abundant classes in the paddy soil were Gammaproteobacteria, Saccharimonadia, Bacteroidia, Alphaproteobacteria, Actinobacteria, and Gemmatimonades with $31.20 \%, 12.39 \%, 12.12 \%, 12.06 \%, 10.04 \%$, and $5.69 \%$, respectively (Figure $3 b$ ). Similarly, among the orders, 12 were significantly more abundant in paddy soil, and eight were more abundant in fallow soil (Supplementary Materials: Figure S1). The significantly higher orders in the paddy soil were Gemmatimonadales, Gaiellales, Anaerolineales, Solirubrobacterales, and Betaproteoles, whereas the significantly higher orders were Sphingoles and Betaproteoles (Supplementary Materials: Figure S1). Among families, 20 were significantly more abundant in the paddy soil, and 12 were significantly more abundant in the fallow soil (Supplementary Materials: Figure S2).

\subsubsection{Bacterial Community Composition of Possible BCAs Genera}

The members of the genera, i.e., Streptomyces, Rhizobium, Bradyrhizobium, Mesorhizobium, Massilia, Bacillus, Paenibacillus, Thiobacillus, and Rhizobacter, have been widely used against rice diseases, thus are regarded as BCAs. The relative abundance of Bacillus, Thiobacillus, Massilia, Methylotenera, Micromonospora, and Pseudonocardia were significantly higher in the fallow soil than in the paddy soil (Figure 4). However, Agromyces, Gemmatimonas, Sphingomonas, Haliangium, and Alterythrobacter were significantly higher in the paddy soil than in the fallow soil. The other possible BCAs, such as Streptomyces, Rhizobium, Bradyrhizobium, Mesorhizobium, and Rhizobacter, were more abundant in the fallow soil than in the paddy soil. Similarly, BCAs such as Pseudomonas, Burkholderia, Lysobacter, Nitrosospira, Haliangium, and Ramlibacter were more abundant in the paddy soil than in the fallow soil. The possible rice pathogens, i.e., Xanthomonas, Pseudoxanthomonas, and Erwinia, were relatively higher in the paddy soil than in the fallow soil (Figure 4).

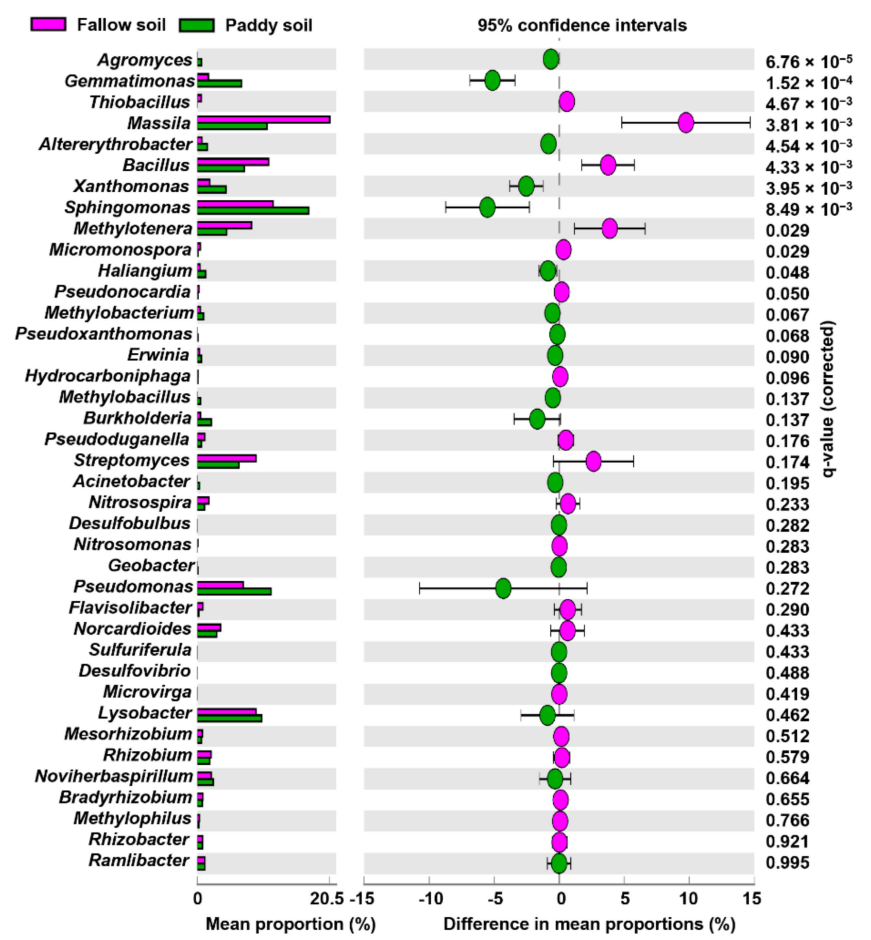

Figure 4. Relative abundances of possible BCAs and pathogenic rice genera. Relative abundance of 39 BCAs and pathogenic rice genera, determined by Welch's $t$-test with a Benjamini-Hochberg FDR test correction to calculate $q$-values. 
3.4. High Abundance of Functional Categories Related to Disease Suppression in the Fallow Soil Than the Paddy Soil

Functional categories, such as molecular communication, plant nutrition, plant adaptability to disease, and other functional categories relevant to growth promotion and plant fitness, were compared between the fallow and paddy soil (Figure 5). In terms of molecular communication, the fallow soil exhibited a significantly higher relative abundance of genes coding for secretion systems and bacterial chemotaxis functions than the paddy soil (Figure 5). In contrast, the paddy soil showed a higher relative abundance of DNA replication, translation, transcription, RNA biosynthesis, and transport genes than the fallow soil. For plant nutrition, nitrogen metabolism genes and plant-pathogen interactions genes were higher in the paddy soil, whereas calcium signaling was significantly higher in the fallow soil. Furthermore, for disease suppression, biosynthesis of siderophores, drug metabolism, toxins, biosynthesis of secondary metabolites, and antibiotics such as streptomycin were significantly higher in the fallow soil than in the paddy soil (Figure 5).

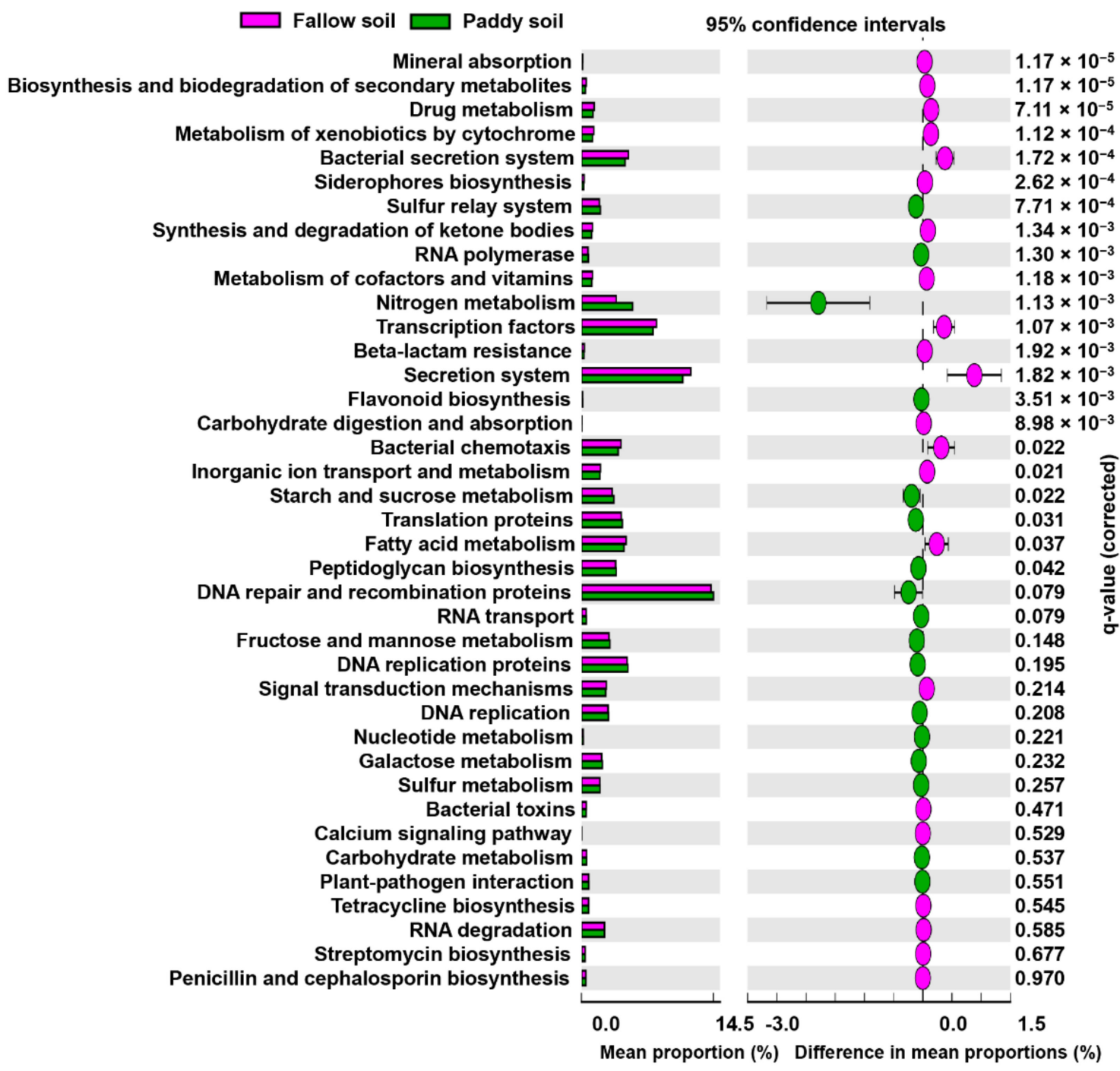

Figure 5. Extended error bar plot illustrating the mean proportion (\%) significantly different functional groups at KEGG level 3. Points represent differences between the fallow and paddy soil (pink and green). Welch's $t$-test was performed with a Benjamini-Hochberg FDR test correction to calculate $q$-values. 


\section{Discussion}

The microbial composition and structure significantly influence soil fertility and health [33,34]. Moreover, soil microorganisms have an essential role in plant health and are considered the second genome of the plants [35]. Therefore, there is a need to understand the microbial composition, diversity, and ecological functions in different soil types to obtain information about the bacterial BCAs and plant pathogens that might influence the life of plants [35].

In our study, the alpha diversity of the bacterial community was reduced in the fallow soil but increased in the paddy soil. Less diversity of the bacterial community in the fallow soil forces us to speculate that it might be due to the failure of many bacterial communities grown under fallow conditions. The increase in the diversity of the bacterial community in the paddy soil may result from the increased levels of fertilizers inputs in the paddy soil than in the fallow soil. Moreover, the fertilizers enhanced the decomposition of organic matter, and the decomposition of organic matter increased microbial diversity [36]. Previous studies reported that this increase in alpha diversity in the paddy soil might also result from higher annual fluxes of organic carbon turnover stemming from plant productivity and litter input [37]. Furthermore, numerous studies also showed that root exudates of rice crops containing acids such as phenolic and organic acids in the paddy soil also affect microbial diversity [38]. Hence the continuous input of organic and inorganic fertilizers and root exudates may have increased the microbial diversity in the paddy soil.

According to earlier studies, these six phyla, i.e., Proteobacteria, Bacteroidetes, Actinobacteria, Chloroflexi, Acidobacteria, and Patescibacteria, are the most dominant and ubiquitous in soil $[39,40]$. Phyla Proteobacteria, Actinobacteria, and Bacteroidetes account for higher abundance in metabolism and genetics, even in other soil types, and most of their members are decomposers [33,41]. Previous studies have also shown that low nutrient levels favor Proteobacteria and Actinobacteria in agroecosystems across the globe. Several genera under these phyla are beneficial and most dominant in the disease suppressive soils [42]. Furthermore, Proteobacteria and Actinobacteria follow the copiotroph/oligotroph hypothesis [43], which postulates that oligotrophic bacteria are more abundant in soil with low nutrient levels. In our study, most likely due to low nutrients in the fallow soil, both phyla follow the copiotroph/oligotroph hypothesis. Moreover, Actinobacteria and Firmicutes are reported to reduce $\mathrm{Fe}^{3+}$ to $\mathrm{Fe}^{2+}$ and generate siderophores and secondary metabolites; all these qualities make these phyla beneficial [44]. In our study, phyla Proteobacteria, Actinobacteria, Bacteroidetes, and Firmicutes exhibited a very high abundance in the fallow soil than the paddy soil, suggesting that fallowing might favor crop growth and health.

The remaining phyla, i.e., Chloroflexi, Nitrospirae, Gemmatimonadetes, Verrucomicrobia, and Patescibacteria, also have a crucial ecological role. Chloroflexi includes facultative anaerobic and heterotrophic oligotrophs genera that can survive on recalcitrant plant polymers [45]. Nitrospirae consists of mostly uncultured nitrite-oxidizing genera. They can mobilize soil $\mathrm{N}$ and enhance its mineralization and availability to plants [46]. The members of Gemmatimonadetes flourish in phosphorus and organic material-rich soils and can modulate carbon and nitrogen [47]. Previous studies revealed that the members of Verrucomicrobia promote growth, colonize rice roots, and play a significant role in the degradation of polysaccharides and carbon cycling [48]. As far as Acidobacteria assemblage is concerned, it is selected explicitly by soil type having high organic matter. This indicates that carbon sources present in paddy soil were a significant factor in increasing the abundance of this phylum [49]. In our study, the phyla Chloroflexi, Verrucomicrobia, Patescibacteria, Gemmatimonadetes, Acidobacteria, and Nitrospirae were observed to be more abundant in the paddy soil than in the fallow soil. Hence, soil conditions cannot be denied and play a significant role in determining the bacterial community in soil types.

Siderophore production and secondary metabolites biosynthesis can be associated with suppressing diseases [50]. 
Most of the species of genera such as Bacillus, Rhizobium, Burkholderia, Bradyrhizobium, Lysobacter, Rhizobacter, and Streptomyces are plant growth promoters and produce siderophores and secondary metabolites to suppress plant diseases. Some of the members of genera, i.e., Bacillus, Bradyrhizobium, and Rhizobium, are also well known for solubilizing phosphates. Moreover, strains of genus Massilia reduce nitrates, suggesting an essential role as plant growth-promoting bacteria [35,44,51]. In addition, members in the genus Rhizobium are well-known, plant-associated nitrogen fixers [52]. In our study, the high relative abundances of possible BCAs and their predicted functions (using tools such as PICRUSt) including siderophore production and biosynthesis of secondary metabolites including streptomycin, an important suppressive antibiotic in fallow soil compared to paddy soil, was a key finding. This might be because most BCAs, especially in the phyla Firmicutes and Actinobacteria, prefer to survive under the fallow soil which has dry soil conditions than in the paddy soil $[42,43]$. Moreover, these phyla capable of utilizing recalcitrant carbon sources are highly present in arid nutrient-poor soil, in our case fallow soil [53,54]. However Proteobacteria has been shown to accumulate in irrigated or non-irrigated soil $[8,54]$.

Xanthomonas oryzae pv. oryzae (Xoo) is widely prevalent in all rice-growing areas of the world and causes Bacterial Leaf Blight [55]. In contrast, Erwinia uredovora is responsible for the discoloration of rice grains [56]. In our study, Xanthomonas and Erwinia were more abundant in the paddy soil. The results of this study can be used as a baseline for further research focusing on BCAs and pathogens in soil types.

\section{Conclusions}

In conclusion, paddy soil has a distinct bacterial diversity and composition than fallow soil. Compared to the paddy soil, the fallow soil microbiome is anticipated to have more functions that support plant growth and suppress plant diseases. BCAs were more abundant in the fallow soil than in the paddy soil. In contrast, the relative abundance of rice pathogens was significantly higher in the paddy soil. A high abundance of BCAs in the fallow soil and fewer pathogens suggested that rice diseases can be minimized by keeping paddy soil fallow for a certain period.

Supplementary Materials: The following are available online at https:/ /www.mdpi.com/article/ 10.3390/agronomy12020431/s1, Figure S1: Significantly abundant orders in the fallow and paddy soils as determined by Welch's $t$-test using the Benjamini-Hochberg $p$-value correction $(p<0.05)$, Figure S2: Significantly abundant families in the fallow and paddy soils as determined by Welch's $t$-test using the Benjamini-Hochberg $p$-value correction $(p<0.05)$, Table S1: Read counts of paddy and fallow soil samples after removal of chloroplast, mitochondria, and potential host DNA, Table S2: The Pairwise Kruskal-Wallis comparisons of alpha diversities in each soil type, Table S3: Permutational MANOVA results using weighted and unweighted UniFrac distances for the soil types.

Author Contributions: Conceptualization, J.D. and A.A.; methodology, A.A. and A.H.A.; software, A.A.; validation, Y.F. and A.A.; formal analysis, A.A.; investigation, A.A. and D.J.; resources, Y.F., Y.L., J.X., J.C. and D.J.; data curation, J.D. and Y.F.; writing-original draft preparation, A.A.; writing-review and editing, J.D. and Y.F.; supervision, J.D.; project administration, J.D.; and funding acquisition, J.D. All authors have read and agreed to the published version of the manuscript.

Funding: This research was funded by China Agriculture Research System of MOF and MARA.

Institutional Review Board Statement: Not applicable.

Informed Consent Statement: Not applicable.

Data Availability Statement: Data sets analyzed during the current study are available from the current author on reasonable request.

Acknowledgments: The first author acknowledges the Chinese Govt Scholarship council (CSC) for providing scholarships for his Ph.D. studies.

Conflicts of Interest: We declare no conflict of interest. 


\section{References}

1. Ding, Y.; Wang, W.; Zhuang, Q.; Luo, Y. Adaptation of paddy rice in China to climate change: The effects of shifting sowing date on yield and irrigation water requirement. Agric. Water Manag. 2020, 228, 105890. [CrossRef]

2. Popp, J.; Harangi-Rákos, M.; Gabnai, Z.; Balogh, P.; Antal, G.; Bai, A. Biofuels and their co-products as livestock feed: Global economic and environmental implications. Molecules 2016, 21, 285. [CrossRef] [PubMed]

3. Ali, M.; Ahmad, Z.; Ashraf, M.F.; Dong, W. Maize endophytic microbial-communities revealed by removing PCR and 16S rRNA sequencing and their synthetic applications to suppress maize banded leaf and sheath blight. Microbiol. Res. 2021, $242,126639$. [CrossRef] [PubMed]

4. Bakker, P.A.H.M.; Pieterse, C.M.J.; de Jonge, R.; Berendsen, R.L. The soil-borne legacy. Cell 2018, 172, 1178-1180. [CrossRef]

5. Alami, M.M.; Xue, J.; Ma, Y.; Zhu, D.; Gong, Z.; Shu, S.; Wang, X. Structure, diversity, and composition of bacterial communities in rhizospheric soil of Coptis chinensis Franch under continuously cropped fields. Diversity 2020, 12, 57. [CrossRef]

6. Somasundaram, J.; Naorem, A.K.; Lal, R.; Dalal, R.C.; Sinha, N.K.; Patra, A.K.; Chaudhari, S.K. Disease-suppressive soils-Beyond food production: A critical review. J. Soil Sci. Plant Nutr. 2021, 21, 1437-1465. [CrossRef]

7. Clairmont, L.K.; Stevens, K.J.; Slawson, R.M. Site-specific differences in microbial community structure and function within the rhizosphere and rhizoplane of wetland plants is plant species dependent. Rhizosphere 2019, 9, 56-68. [CrossRef]

8. Huang, M.; Tian, A.; Chen, J.; Cao, F.; Chen, Y.; Liu, L. Soil bacterial communities in three rice-based cropping systems differing in productivity. Sci. Rep. 2020, 10, 9867. [CrossRef]

9. Hacquard, S.; Garrido-Oter, R.; González, A.; Spaepen, S.; Ackermann, G.; Lebeis, S.; McHardy, A.C.; Dangl, J.L.; Knight, R.; Ley, R.; et al. Microbiota and host nutrition across plant and animal kingdoms. Cell Host Microbe 2015, 17, 603-616. [CrossRef]

10. Castrillo, G.; Teixeira, P.J.P.L.; Paredes, S.H.; Law, T.F.; de Lorenzo, L.; Feltcher, M.E.; Finkel, O.M.; Breakfield, N.W.; Mieczkowski, P.; Jones, C.D.; et al. Root microbiota drive direct integration of phosphate stress and immunity. Nature 2017, 543, 513-518. [CrossRef]

11. Fitzpatrick, C.R.; Copeland, J.; Wang, P.W.; Guttman, D.S.; Kotanen, P.M.; Johnson, M.T.J. Assembly and ecological function of the root microbiome across angiosperm plant species. Proc. Natl. Acad. Sci. USA 2018, 115, E1157-E1165. [CrossRef]

12. Shrestha, B.K.; Karki, H.S.; Groth, D.E.; Jungkhun, N.; Ham, J.H. Biological control activities of rice-associated Bacillus sp. strains against sheath blight and bacterial panicle blight of rice. PLoS ONE 2016, 11, e0146764. [CrossRef]

13. Shanmugaiah, V.; Mathivanan, N.; Varghese, B. Purification, crystal structure and antimicrobial activity of phenazine-1carboxamide produced by a growth-promoting biocontrol bacterium, Pseudomonas aeruginosa MML2212. J. Appl. Microbiol. 2010, 108, 703-711. [CrossRef]

14. Yang, J.H.; Liu, H.X.; Zhu, G.M.; Pan, Y.L.; Xu, L.P.; Guo, J.H. Diversity analysis of antagonists from rice-associated bacteria and their application in biocontrol of rice diseases. J. Appl. Microbiol. 2008, 104, 91-104. [CrossRef]

15. Yang, J.H.; Zhang, W.W.; Zhuang, Y.Q.; Xiao, T. Biocontrol activities of bacteria from cowdung against the rice sheath blight pathogen. J. Plant Dis. Prot. 2017, 124, 131-141. [CrossRef]

16. Lazcano, C.; Boyd, E.; Holmes, G.; Hewavitharana, S.; Pasulka, A.; Ivors, K. The rhizosphere microbiome plays a role in the resistance to soil-borne pathogens and nutrient uptake of strawberry cultivars under field conditions. Sci. Rep. 2021, 11, 3188. [CrossRef]

17. Derakhshani, H.; Tun, H.M.; Khafipour, E. An extended single-index multiplexed 16S rRNA sequencing for microbial community analysis on MiSeq illumina platforms. J. Basic Microbiol. 2016, 56, 321-326. [CrossRef]

18. Caporaso, J.G.; Kuczynski, J.; Stombaugh, J.; Bittinger, K.; Bushman, F.D.; Costello, E.K.; Fierer, N.; Peña, A.G.; Goodrich, J.K.; Gordon, J.I.; et al. QIIME allows analysis of high-throughput community sequencing data. Nat. Methods 2010, 7, 335-336. [CrossRef]

19. Callahan, B.J.; McMurdie, P.J.; Rosen, M.J.; Han, A.W.; Johnson, A.J.A.; Holmes, S.P. DADA2: High-resolution sample inference from Illumina amplicon data. Nat. Methods 2016, 13, 581-583. [CrossRef]

20. Quast, C.; Pruesse, E.; Yilmaz, P.; Gerken, J.; Schweer, T.; Yarza, P.; Peplies, J.; Glöckner, F.O. The SILVA ribosomal RNA gene database project: Improved data processing and web-based tools. Nucleic Acids Res. 2013, 41, D590-D596. [CrossRef]

21. Fisher, R.A.; Corbet, A.S.; Williams, C.B. The relation between the number of species and the number of individuals in a random sample of an animal population. J. Anim. Ecol. 1943, 12, 42-58. [CrossRef]

22. Shannon, C.E. A Mathematical theory of communication. Bell Syst. Tech. J. 1948, 27, 379-423. [CrossRef]

23. Pielou, E.C. The measurement of diversity in different types of biological collections. J. Theor. Biol. 1966, 13, 131-144. [CrossRef]

24. Barker, G.M. Phylogenetic diversity: A quantitative framework for measurement of priority and achievement in biodiversity conservation. Biol. J. Linn. Soc. 2008, 76, 165-194. [CrossRef]

25. Böhning, D. Chao's lower bound estimator and the size of the Pleiades. Environ. Ecol. Stat. 2020, 27, 171-173. [CrossRef]

26. Lozupone, C.; Lladser, M.E.; Knights, D.; Stombaugh, J.; Knight, R. UniFrac: An effective distance metric for microbial community comparison. ISME J. 2011, 5, 169-172. [CrossRef]

27. Vázquez-Baeza, Y.; Pirrung, M.; Gonzalez, A.; Knight, R. EMPeror: A tool for visualizing high-throughput microbial community data. Gigascience 2013, 2, 16. [CrossRef]

28. Langille, M.G.I.; Zaneveld, J.; Caporaso, J.G.; McDonald, D.; Knights, D.; Reyes, J.A.; Clemente, J.C.; Burkepile, D.E.; Vega Thurber, R.L.; Knight, R.; et al. Predictive functional profiling of microbial communities using $16 \mathrm{~S}$ rRNA marker gene sequences. Nat. Biotechnol. 2013, 31, 814-821. [CrossRef] 
29. DeSantis, T.Z.; Hugenholtz, P.; Larsen, N.; Rojas, M.; Brodie, E.L.; Keller, K.; Huber, T.; Dalevi, D.; Hu, P.; Andersen, G.L. Greengenes, a chimera-checked $16 \mathrm{~S}$ rRNA gene database and workbench compatible with ARB. Appl. Environ. Microbiol. 2006, 72, 5069-5072. [CrossRef]

30. Parks, D.H.; Tyson, G.W.; Hugenholtz, P.; Beiko, R.G. STAMP: Statistical analysis of taxonomic and functional profiles. Bioinformatics 2014, 30, 3123-3124. [CrossRef]

31. Benjamini, Y.; Hochberg, Y. Controlling the false discovery rate: A practical and powerful approach to multiple testing. J. R. Stat. Soc. Ser. B 1995, 57, 289-300. [CrossRef]

32. Anderson, M.J. Permutational Multivariate Analysis of Variance (PERMANOVA). In Wiley StatsRef: Statistics Reference Online; Balakrishnan, N., Colton, T., Everitt, B., Piegorsch, W., Ruggeri, F., Teugels, J.L., Eds.; John Wiley \& Sons, Ltd.: Hoboken, NJ, USA, 2017; pp. 1-15. [CrossRef]

33. Curd, E.E.; Martiny, J.B.H.; Li, H.; Smith, T.B. Bacterial diversity is positively correlated with soil heterogeneity. Ecosphere 2018, 9 , e02079. [CrossRef]

34. Wang, R.; Xiao, Y.; Lv, F.; Hu, L.; Wei, L.; Yuan, Z.; Lin, H. Bacterial community structure and functional potential of rhizosphere soils as influenced by nitrogen addition and bacterial wilt disease under continuous sesame cropping. Appl. Soil Ecol. 2018, 125, 117-127. [CrossRef]

35. Berendsen, R.L.; Pieterse, C.M.; Bakker, P.A. The rhizosphere microbiome and plant health. Trends Plant Sci. 2012, 17, 478-486. [CrossRef]

36. Cui, X.; Zhang, Y.; Gao, J.; Peng, F.; Gao, P. Long-term combined application of manure and chemical fertilizer sustained higher nutrient status and rhizospheric bacterial diversity in reddish paddy soil of Central South China. Sci. Rep. 2018, 8, 16554 [CrossRef]

37. Mackelprang, R.; Grube, A.M.; Lamendella, R.; da Conceição Jesus, E.; Copeland, A.; Liang, C.; Jackson, R.D.; Rice, C.W.; Kapucija, S.; Parsa, B.; et al. Microbial community structure and functional potential in cultivated and native tallgrass prairie soils of the Midwestern United States. Front. Microbiol. 2018, 9, 1775. [CrossRef]

38. Xiong, L.; Liu, X.; Vinci, G.; Spaccini, R.; Drosos, M.; Li, L.; Piccolo, A.; Pan, G. Molecular changes of soil organic matter induced by root exudates in a rice paddy under $\mathrm{CO}_{2}$ enrichment and warming of canopy air. Soil Biol. Biochem. 2019, 137, 107544. [CrossRef]

39. Delgado-Baquerizo, M.; Oliverio, A.M.; Brewer, T.E.; Benavent-González, A.; Eldridge, D.J.; Bardgett, R.D.; Maestre, F.T.; Singh, B.K.; Fierer, N. A global atlas of the dominant bacteria found in soil. Science 2018, 359, 320-325. [CrossRef]

40. Janssen, P.H. Identifying the dominant soil bacterial taxa in libraries of $16 \mathrm{~S}$ rRNA and 16S rRNA genes. Appl. Environ. Microbiol. 2006, 72, 1719-1728. [CrossRef]

41. Zhan, Y.; Liu, W.; Bao, Y.; Zhang, J.; Petropoulos, E.; Li, Z.; Lin, X.; Feng, Y. Fertilization shapes a well-organized community of bacterial decomposers for accelerated paddy straw degradation. Sci. Rep. 2018, 8, 7981. [CrossRef]

42. Dai, Z.; Su, W.; Chen, H.; Barberán, A.; Zhao, H.; Yu, M.; Yu, L.; Brookes, P.C.; Schadt, C.W.; Chang, S.X.; et al. Long-term nitrogen fertilization decreases bacterial diversity and favors the growth of Actinobacteria and Proteobacteria in agroecosystems across the globe. Glob. Change Biol. 2018, 24, 3452-3461. [CrossRef]

43. Fierer, N.; Bradford, M.A.; Jackson, R.B. Toward an ecological classification of soil bacteria. Ecology 2007, 88, 1354-1364. [CrossRef]

44. Lee, S.M.; Kong, H.G.; Song, G.C.; Ryu, C.M. Disruption of Firmicutes and Actinobacteria abundance in tomato rhizosphere causes the incidence of bacterial wilt disease. ISME J. 2021, 15, 330-347. [CrossRef]

45. King, G.M. Urban microbiomes and urban ecology: How do microbes in the built environment affect human sustainability in cities? J. Microbiol. 2014, 52, 721-728. [CrossRef]

46. Palomo, A.; Fowler, S.J.; Gülay, A.; Rasmussen, S.; Sicheritz-Ponten, T.; Smets, B.F. Metagenomic analysis of rapid gravity sand filter microbial communities suggests novel physiology of Nitrospira spp. ISME J. 2016, 10, 2569-2581. [CrossRef]

47. Su, J.Q.; Ding, L.J.; Xue, K.; Yao, H.Y.; Quensen, J.; Bai, S.J.; Wei, W.X.; Wu, J.S.; Zhou, J.; Tiedje, J.M.; et al. Long-term balanced fertilization increases the soil microbial functional diversity in a phosphorus-limited paddy soil. Mol. Ecol. 2015, 24, 136-150. [CrossRef]

48. Bünger, W.; Jiang, X.; Müller, J.; Hurek, T.; Reinhold-Hurek, B. Novel cultivated endophytic Verrucomicrobia reveal deep-rooting traits of bacteria to associate with plants. Sci. Rep. 2020, 10, 8692. [CrossRef]

49. Kielak, A.M.; Barreto, C.C.; Kowalchuk, G.A.; van Veen, J.A.; Kuramae, E.E. The ecology of Acidobacteria: Moving beyond genes and genomes. Front. Microbiol. 2016, 7, 744. [CrossRef]

50. Lutz, S.; Thuerig, B.; Oberhaensli, T.; Mayerhofer, J.; Fuchs, J.G.; Widmer, F.; Freimoser, F.M.; Ahrens, C.H. Harnessing the microbiomes of suppressive composts for plant protection: From metagenomes to beneficial microorganisms and reliable diagnostics. Front. Microbiol. 2020, 11, 1810. [CrossRef]

51. Zhong, W.; Gu, T.; Wang, W.; Zhang, B.; Lin, X.; Huang, Q.; Shen, W. The effects of mineral fertilizer and organic manure on soil microbial community and diversity. Plant Soil 2010, 326, 511-522. [CrossRef]

52. Tang, Y.; Yu, G.; Zhang, X.; Wang, Q.; Ge, J.; Liu, S. Changes in nitrogen-cycling microbial communities with depth in temperate and subtropical forest soils. Appl. Soil Ecol. 2018, 124, 218-228. [CrossRef]

53. Mohammadipanah, F.; Wink, J. Actinobacteria from arid and desert habitats: Diversity and biological activity. Front. Microbiol. 2016, 6, 1541. [CrossRef] [PubMed]

54. Bachar, A.; Al-Ashhab, A.; Soares, M.I.M.; Sklarz, M.Y.; Angel, R.; Ungar, E.D.; Gillor, O. Soil microbial abundance and diversity along a low precipitation gradient. Microb. Ecol. 2010, 60, 453-461. [CrossRef] [PubMed] 
55. Yasmin, S.; Hafeez, F.Y.; Mirza, M.S.; Rasul, M.; Arshad, H.M.I.; Zubair, M.; Iqbal, M. Biocontrol of bacterial leaf blight of rice and profiling of secondary metabolites produced by rhizospheric Pseudomonas aeruginosa brp3. Front. Microbiol. 2017, 8, 1895. [CrossRef]

56. Yan, H.; Yu, S.H.; Xie, G.L.; Fang, W.; Su, T.; Li, B. Grain discoloration of rice caused by Pantoea ananatis (synonym Erwinia uredovora) in China. Plant Dis. 2010, 94, 482. [CrossRef] 